\title{
Nonlinear Resonant Tunneling through an Anderson Impurity at Low Temperature
}

\author{
T. K. Ng \\ Department of Physics, Hong Kong Unicersity of Science and Technology, Clear Water Bay Road, Kowloon, Hong Kong
}

(Received 21 January 1993)

\begin{abstract}
The problem of nonlinear resonant tunneling through an Anderson impurity is investigated in this Letter using a combined approach of Keldysh formalism and $1 / N$ expansion at zero temperature. We find that to order $1 / N$, the Kondo resonance is not destroyed by any finite potential difference between external probes. The resulting one-electron Green's functions and differential conductance are presented. The effect of finite temperature is discussed.
\end{abstract}

PACS numbers: 72.15.Qm, 73.20.Dx, 73.40.Gk, 73.50.Fq

Recently there is much interest in the study of transports in mesoscopic systems with strong electron-electron correlations $[1-10]$. It is believed that these systems constitute a new area of research where novel phenomena associated with quantum coherence in the presence of electron-electron interaction can be probed. Among others, a problem of particular interest is resonant tunneling through an Anderson impurity in the nonlinear transport regime [9-11]. In the linear transport regime, it is understood that the Kondo effect occurs in this system leading to enhancement in the transmittance of electrons tunneling from one probe to another at temperature $T<T_{K}$ ( $T_{K}=$ Kondo temperature) $[5,6]$. The interesting problem is whether in the nonlinear regime, where the potential difference between external probes can be larger than $T_{K}$, a Kondo type effect can still occur. The problem constitutes a unique example where the effect of nonequilibrium (finite potential difference) on a nontrivial quantum coherent system can be studied.

We shall study the problem using the model Hamiltonian

$$
\begin{aligned}
H= & \sum_{k, \sigma, \eta=L, R} \varepsilon_{k} c_{k n \sigma}^{\dagger} c_{k \eta \sigma}+\sum_{\sigma} \varepsilon_{o} c_{\sigma}^{\dagger} c_{\sigma}+U_{n \nmid n \downarrow} \\
& +\sum_{k, \sigma, \eta=L, R}\left(V_{\eta} c_{k \eta \sigma}^{\dagger} c_{\sigma}+\text { H.c. }\right),
\end{aligned}
$$

where $c_{k \eta \sigma}^{\dagger}\left(c_{k \eta \sigma}\right)$ creates (destroys) an electron with momentum $k$ and spin $\sigma$ in the lead $\eta(=L, R)$, and $c_{\sigma}^{\dagger}$ $\left(c_{\sigma}\right)$ creates (destroys) a spin- $\sigma$ electron on the impurity site $\varepsilon_{o} . U$ is an on-site Coulomb interaction. Notice that this model Hamiltonian in fact simulates the physical situation of a semiconductor quantum dot weakly coupled to two external reservoirs with $\varepsilon_{o}$ being a quantized level in the quantum dot [4]. The new feature here is that the chemical potentials $\mu_{L}$ and $\mu_{R}$ in the two leads may not be equal.

We shall study the problem at zero temperature using a combination of Keldysh technique and variational wave-function calculation ( $1 / N$ expansion). The Keldysh technique is a generalization of the usual many-body approach to nonequilibrium situations [12-14] where the interaction $U$ or hopping $V_{\eta}$ onto the $\varepsilon_{o}$ site is turned on adiabatically as a perturbation $[10,14]$. In the steady state, the system is characterized by two Green's functions [10], $G_{o o}^{R}(\omega)$ and $G_{o o}^{<}(\omega)$, where $G^{R}$ and $G^{<}$are Fourier transforms of the retarded Green's functions

$$
G_{o o}^{R}\left(t-t^{\prime}\right)=-i \theta\left(t-t^{\prime}\right)\left\{\left\langle c_{\sigma}(t) c_{\sigma}^{\dagger}\left(t^{\prime}\right)\right\rangle+\left\langle c_{\sigma}^{\dagger}\left(t^{\prime}\right) c_{\sigma}(t)\right\rangle\right\}
$$

and $G_{o o}^{<}\left(t-t^{\prime}\right)=-i\left\langle c_{\sigma}^{\dagger}(t) c_{\sigma}\left(t^{\prime}\right)\right\rangle$, respectively. $G_{o o}^{<}(\omega)$ measures the density of occupied states at energy $\omega[10]$. In the flatband limit, $G^{R}$ and $G^{<}$are related to the irreducible retarded self-energy $\Sigma^{R}(\omega)$ and "scattering-in" self-energy $\Sigma<(\omega)$ by [10]

$$
G_{o o}^{R}(\omega)=\frac{1}{\omega-\varepsilon_{o}+i \Gamma-\Sigma^{R}(\omega)}
$$

and

$$
\begin{aligned}
G_{o o}^{<}(\omega)=G_{o o}^{R}(\omega) G_{o o}^{A}(\omega)[ & -\Sigma<(\omega)+2 i \Gamma_{L} n_{L}(\omega) \\
& \left.+2 i \Gamma_{R} n_{R}(\omega)\right]
\end{aligned}
$$

where $\Gamma=\Gamma_{L}+\Gamma_{R}, \Gamma_{\eta}=N(0) V_{\eta}^{2}$ is the tunneling rate of the electron from site $\varepsilon_{o}$ to reservoir $\eta$ and $n_{\eta}(\omega)$ $=\left\{\exp \left[\beta\left(\omega-\mu_{\eta}\right)\right]+1\right\}^{-1}$ is the occupation of the electrons in the corresponding resevior. $G^{A}=G^{R *}$ is the advanced Green's function. Notice that all the statistical information (i.e., information of the unperturbed state) is contained in $n_{\eta}(\omega)$ whereas the dynamical information of the perturbation is contained in the two self-energies. Notice that for the nonequilibrium situation $\mu_{L} \neq \mu_{R}$, the imaginary part of the self-energy $\Sigma^{R}(\omega)$ is in general not equal to zero on the Fermi surface even at zero temperature as can be checked by perturbation theory for small $U$ [10]. However, the sharp Fermi surface structure in $n_{\eta}(\omega)$ (at zero temperature) remains in $G_{o o}^{<}(\omega)[10]$ in contrast to the equilibrium situation. This nontrivial behavior is a direct consequence of the fact that statistical and dynamical information for systems described by a general density matrix are decoupled in general [12,13]. The two properties of the system are related to each other only in the equilibrium situation through thermodynamics.

The existence of the sharp Fermi surface for general $\mu_{L} \neq \mu_{R}$ leads to an interesting possibility that in the large $U$ limit and with $\varepsilon_{o} \ll \min \left(\mu_{L}, \mu_{R}\right)$, the Kondo effect occurring at the equilibrium situation which is a conse- 
quence of the sharp Fermi surface may still remain for $\mu_{L} \neq \mu_{R}$. We shall study this possibility in the following using the $1 / N$ expansion technique for the infinite- $U$ situation which is known to work well in equilibrium [15]. We shall carry out the calculation to order $O(1 / N)$.

We shall consider temperature $T=0$ and work with the variation wave-function approach to $1 / N$ expansion ( $N$ is the number of spin components, $N=2$ in the physical situation) modified for the nonequilibrium situation. To order $1 / N$, our variational wave function is of the form $[15,16]$

$$
|\Psi\rangle=A\left\{|\Omega\rangle+\sum_{k, \eta, \sigma}\left[\alpha_{\eta k}\left|\varepsilon_{o} \varepsilon_{\eta k} \sigma\right\rangle+\sum_{k^{\prime}, \eta^{\prime}}\left(\beta_{k \eta k^{\prime} \eta^{\prime}}\left|E_{\eta^{\prime} k^{\prime}} \varepsilon_{\eta k} \sigma\right\rangle+\sum_{k^{\prime \prime}, \eta^{\prime \prime}, \sigma^{\prime}} \gamma_{\eta^{\prime \prime} k^{\prime \prime} \eta^{\prime} k^{\prime} \eta k}\left|\left(\varepsilon_{o} \varepsilon_{\eta^{\prime \prime} k^{\prime \prime}} \sigma\right)\left(E_{\eta^{\prime} k^{\prime}} \varepsilon_{\eta k} \sigma^{\prime}\right)\right\rangle\right)\right]\right\},
$$

where

$$
\left|\varepsilon_{o} \varepsilon_{\eta k} \sigma\right\rangle=c_{o}^{\dagger} c_{\eta k \sigma}|\Omega\rangle,\left|E_{\eta^{\prime} k^{\prime}} \varepsilon_{\eta k} \sigma\right\rangle=c_{\eta^{\prime} k^{\prime} \sigma}^{\dagger} c_{\eta k \sigma}|\Omega\rangle
$$

and

$$
\left.\left|\left(\varepsilon_{o} \varepsilon_{\eta^{\prime \prime} k^{\prime \prime}} \sigma\right)\left(E_{\eta^{\prime} k^{\prime}} \varepsilon_{\eta k} \sigma^{\prime}\right)\right\rangle=c_{\sigma}^{\dagger} c_{\eta^{\prime \prime} k " \sigma} c_{\eta^{\prime}}^{\dagger} c_{\eta^{\prime} k^{\prime} \sigma^{\prime} c_{\eta k \sigma^{\prime}}|\Omega\rangle}\right\rangle
$$

$|\Omega\rangle$ represents a state with filled left and right Fermi seas with chemical potentials $\mu_{L}$ and $\mu_{R}$, respectively, and with an empty $\varepsilon_{o}$ level. We shall assume $\mu_{L}>\mu_{R}$ in the following discussions unless specified. $A$ is a wavefunction renormalization constant and $\alpha, \beta, \gamma$ are variational parameters. The first two terms in the wave function are of order $O(1)$ in $1 / N$ expansion whereas the last two terms are of order $O(1 / N)[15,16]$. Notice that $|\Psi\rangle$ is a spin-singlet wave function by construction. To order $O(1 / N)$, variation results in a self-consistent equation for the ground-state energy

$$
\begin{aligned}
E_{G}= & 2 \Gamma_{L} \int_{-D}^{D} d \varepsilon n_{L}(\varepsilon) G\left(E_{G}+\varepsilon\right) \\
& +2 \Gamma_{R} \int_{-D}^{D} d \varepsilon n_{R}(\varepsilon) G\left(E_{G}+\varepsilon\right),
\end{aligned}
$$

where $G(z)=1 /\left[z-\varepsilon_{o}-\Sigma_{o}(z)\right]$ is the propagator describing an occupied $\varepsilon_{o}$ site [15]. $\Sigma_{o}(z)$ is a self-energy of order $1 / N$ coming from one particle-hole pair excitations from the Fermi seas. 2D is the bandwidth of the reservoirs. The factor of 2 is coming from summing over spins. We shall not discuss $\Sigma_{o}(z)$ in detail here except to point out that the particle and hole are not restricted to coming from the same Fermi sea in $\Sigma_{o}$. Configurations where the particle is coming from one Fermi sea and the hole from the other Fermi sea are allowed. In particular, in the nonequilibrium situation $\mu_{L} \neq \mu_{R}$, decay of a particle (or hole) on the Fermi surface after tunneling to the opposite Fermi sea is allowed in our wave function (3).

To order $O(1)$ (i.e., neglecting $\Sigma_{o}$ ), it is easy to see that there exist two solutions to Eq. (4), with $E_{G} \sim\left(\varepsilon_{o}\right.$ $\left.-\mu_{\eta}-T_{K \eta}\right), \eta=L, R$, corresponding to the Kondo effect associated separately with the left and right Fermi seas, respectively. Assuming $\Delta \mu=\mu_{L}-\mu_{R} \gg T_{K}$, the lower energy (and hence ground state) solution corresponds to pinning of the Kondo resonance to the left Fermi sea with $T_{K} \sim D(D / \Delta \mu) g \exp \left[\pi\left(\varepsilon_{o}-\mu_{L}\right) / 2 \Gamma_{L}\right]$ where $g \sim \Gamma_{R} / \Gamma_{L}$. Notice that to this order of approximation, the groundstate wave function has the Kondo resonance pinned at only one Fermi surface. Resonance with the other Fermi surface is not observed in the ground state. We shall see later that both resonances are in fact observable through the Green's functions $G^{R}$ and $G^{<}$which measure the excitation spectrum of the system. The other resonance appears as a collective excitation.

Inclusion of the $1 / N$ self-energy $\Sigma_{o}(z)$ does not modify the above physical picture qualitatively except that besides the above solution, there appears another lower energy solution with $E_{G} \sim\left[\varepsilon_{o}-\mu_{L}-\left(\mu_{L}-\mu_{R}\right)-T_{K}\right]$. Physically, besides having a Kondo resonance pinning to the left Fermi sea, the energy of the system can be lowered further by moving another electron from the left Fermi surface to the right Fermi surface. This extra possibility reflects the fact that the system we are considering is in fact not in an equilibrium state, and the energy can be lowered by simply transferring electrons to achieve equilibrium. However, what is more interesting is that the original nonequilibrium steady state solution we find at $O(1)$ still remains at order $O(1 / N)$ (with $T_{K}$ slightly modified), indicating that the solution in fact represents a stable, nonequilibrium steady state of the system in the prescence of dissipation. We shall analyze this steady state solution in the following.

The Green's functions $G^{R}$ and $G^{<}$for a spin- $\sigma$ electron are given by $[15,16]$

$$
\begin{aligned}
G_{o o}^{R}(\omega)= & \left\langle\Psi\left|c_{\sigma}^{\dagger} \frac{1}{\omega+i \delta-E_{G}+H} c_{\sigma}\right| \Psi\right\rangle \\
& +\left\langle\Psi\left|c_{\sigma} \frac{1}{\omega+i \delta+E_{G}-H} c_{\sigma}^{\dagger}\right| \Psi\right\rangle \\
= & G_{o o}^{-}(\omega)+G_{o o}^{+}(\omega),
\end{aligned}
$$

and

$$
G_{o o}^{<}(\omega)=-(i / \pi) \operatorname{Im} G_{o o}^{-}(\omega) .
$$

To compute these Green's functions, one has to compute the resolvents $\left(z \mp E_{G} \pm H\right)^{-1}$ sandwiched between our variational wave function $|\Psi\rangle$ approximately. The resolvents can be computed in a $1 / N$ expansion by inverting the operators $\left(z \mp E_{G} \pm H\right)$ in a suitably restricted subspace $[15,16]$. The self-energy $\Sigma^{R}(\omega)$ can be obtained by looking at $G_{o o}^{R}(\omega)^{-1}$ afterward. The details of these calculations will be presented in a later paper. We shall summarize our findings here.

First of all, we find that as discussed above, the Fermi edge structure in $G_{o o}<(\omega)$ remains although $\operatorname{Im} \Sigma^{R}(\omega) \neq 0$, with $G^{R} G^{A} \sim Z_{g}^{2} /\left(\omega-\mu_{L}-T_{K}\right)^{2}$ in Eq. (2b), where $Z_{g}$ 
$\sim T_{K} / \Gamma$ is the wave-function renormalization constant for the ground state. The discontinuity is of order $O(1)$ on the left Fermi surface, and is of order $O\left(T_{K} / \Delta \mu\right)^{2}$ on the right. The imaginary part of $\Sigma^{R}(\omega)$ has the following properties around the two Fermi surfaces. For $\Delta \mu<T_{K}$, we find that $\operatorname{Im} \Sigma^{R}(\omega)=0$ for $\mu_{L}-T_{K}<\omega<\mu_{R}+T_{K}$. The Fermi liquid lifetime $\operatorname{Im} \Sigma^{R}(\omega) \sim(\Delta \mu)^{2}$ [10] is found to be missing in our $O(1 / N)$ calculation. For $\Delta \mu>T_{K}$, we find that $\operatorname{Im} \Sigma^{R}\left(\mu_{R}\right)>\operatorname{Im} \Sigma^{R}\left(\mu_{L}\right) \sim\left(\Delta \mu-T_{K}\right)$, indicating that the dynamics for the particle decay in this regime is non-Fermi-liquid-like. This is not surprising since it is well known that in the equilibrium situation, Fermi liquid behavior is expected only at $|\omega-\mu|<T_{K}$. The appearance of finite $\operatorname{Im} \Sigma^{R}(\omega)$ on the Fermi surfaces for $\Delta \mu>T_{K}$ indicates that in this regime, the Kondo peaks will be broadened by inelastic effects (with the lower energy Kondo peak broadened more), and the broadening increases with increasing $\Delta \mu$. To examine the Kondo peaks, we compute the spectral function $\dot{S}(\omega)=-(1 / \pi)$ $\times \operatorname{Im} G_{o o}^{R}(\omega)$ to order $O(1 / N)$ and the results for three different values of $\Delta \mu$ are shown in Fig. 1. We have taken $\Gamma_{L}=\Gamma_{R}=0.5, D=100, \varepsilon_{o}=-5$, and $\mu_{R}=-\mu_{L}$ in our calculations. The Kondo temperature at equilibrium is $T_{K 0}=0.036$ for this set of parameters. To order $O(1)$, the only feature in $S$ is $S(\omega)=Z_{g} \delta\left(\omega-\mu_{L}-T_{K}\right)$, corresponding to a sharp Kondo resonance at $\omega=\mu_{L}+T_{K}$. This feature persists to order $O(1 / N)$, resulting in the nonanalytic behavior of $S(\omega)$ around $\omega \sim \mu_{L}+T_{K}$ in Fig. 1. The reason for this nonanalytic behavior in the $1 / N$ expansion is that the lifetime effects in $S, i \Gamma-i$ $\times \operatorname{Im} \Sigma^{R}(\omega)$, is of order $O(1 / N)$ in the $1 / N$ expansion [15]. Thus a straight forward expansion of $S(\omega)$ to order $O(1 / N)$ will have the singularity remaining around $\omega \sim \mu_{L}+T_{K}$ (except that the values of $Z_{g}$ and $T_{K}$ are renormalized). The singularity can be removed only if one

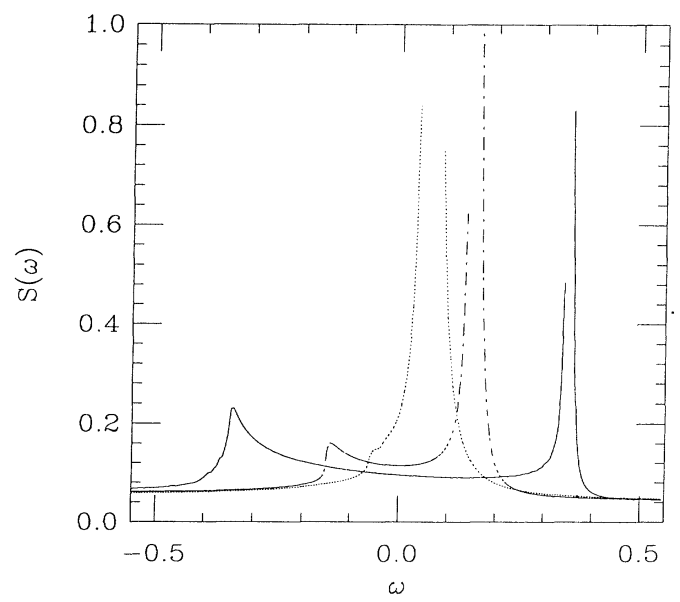

FIG. 1. Spectral functions of the retarded on-site Green's function for three different values of $\Delta \mu: \Delta \mu=0.1$ (dotted line), $\Delta \mu=0.3$ (dot-dashed line), and $\Delta \mu=0.7$ (solid line). The other parameters are chosen as stated in the text. performs an infinite order resummation of the $1 / N$ series, as in the case of noncrossing approximation [15]. Nevertheless, the splitting of the left and right Kondo peaks as $\Delta \mu$ increases is clear in our figure. Notice that the left Kondo peak is getting narrower $\left(T_{K}\right.$ decreases $)$ and the spectral weight is shifted to the right Kondo peak as $\Delta \mu$ increases. Notice also that a rather large amount of spectral weight is still concentrated at the region $\mu_{R}<\omega$ $\left\langle\mu_{L}\right.$, even for $\Delta \mu \gg T_{K}$, indicating that quantum coherence around the Fermi surface persists even though the Kondo peaks are largely broadened by the inelastic effect.

To examine the Kondo effect further, we study the current-voltage relation of the system. The current $I$ is given by [17]

$$
I=2 \frac{e}{h} \Gamma_{\text {red }} \int_{-D}^{D} d \omega\left[n_{L}(\omega)-n_{R}(\omega)\right] S(\omega),
$$

where $\Gamma_{\text {red }}=\Gamma_{L} \Gamma_{R} / \Gamma$. The factor of 2 is coming from spin summation. It is clear that at zero temperature, $I$ is a measure of the total spectral weight sandwiched between $\mu_{R}$ and $\mu_{L}$. Our results are shown in Fig. 2 where we have plotted the differential conductance $d I / d \mu$ as a function of $\mu_{L}$ for fixed $\mu_{R}=0$. The other parameters are chosen as before. Notice that only one single peak centered at $\mu_{L}=\mu_{R}$ with the half-width $\sim T_{K 0}$ is observed in the differential conductance. This is because the Kondo resonances are always pinned at Fermi surfaces and with decreasing strength as $\Delta \mu$ increases. As temperature increases, the peak in $d I / d \mu$ decreases gradually with the characteristic temperature $\sim T_{K 0}$ without broadening (finite temperature properties of the system are studied by a combination of high temperature expansion and the equation of motion method [4]). Notice that the characteristic Kondo temperatures at different $\Delta \mu$ 's are in fact different. Taking $\Gamma_{L} \sim \Gamma_{R}$ and assuming $\Delta \mu \gg T_{K 0}$, we find that $T_{K}\left(\mu_{L}\right) \sim\left(T_{K 0} / \Delta \mu\right) T_{K 0}$. The decreasing $T_{K}$

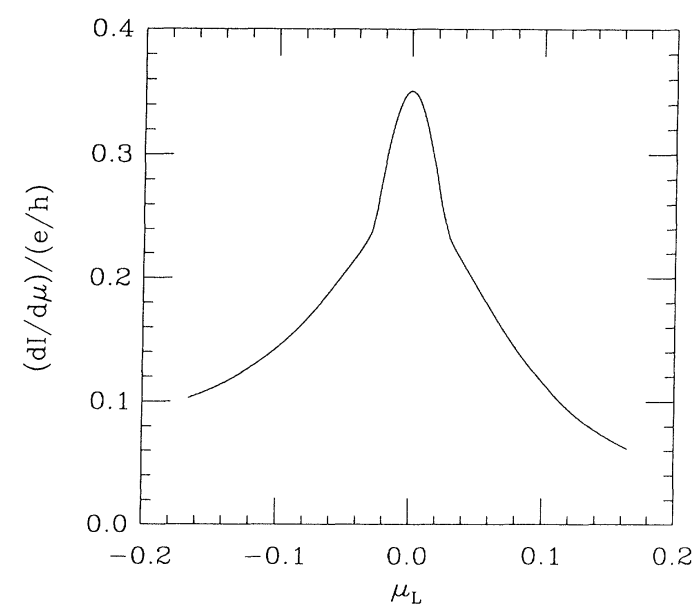

FIG. 2. Differential conductance $d I / d \mu$, with $\mu_{R}=0$, vs applied bias $\left(\mu_{L}\right)$ at zero temperature. Notice that the line shape is non-Lorentzian and asymmetric with respect to $\mu_{L}=0$. 
with increasing $\Delta \mu$ implies that for fixed finite temperature $T \lesssim T_{K 0}$, the Kondo peaks will get weakened and disappear as $\Delta \mu$ is large enough. Unfortunately this effect does not have a strong effect on $I-V$ characteristics since contributions away from the Kondo peaks become dominant as $\Delta \mu$ increases.

We now discuss the physical reason why the Kondo effect can exist in the nonequilibrium situation in the Keldysh approach. It is convenient to imagine the coupling of the $\varepsilon_{o}$ state to reservoirs $V_{\eta}$ 's as perturbations in our variational approach. At zero temperature the unperturbed state consists of two filled Fermi seas with an empty $\varepsilon_{o}$ site. Turning on the perturbation adiabatically changes the wave function continuously. As long as the perturbation introduces no divergence or instability, no other states will be generated and the resulting wave function will still be a quantum coherent spin-singlet state. Notice, however, that since we are in a nonequilibrium situation, the resulting spin-singlet state may have a character different from the equilibrium Kondo state. In fact, the Kondo resonance behavior is found to be very different in a small $U$ expansion treatment of the symmetric Anderson model out of equilibrium [10]. In our $1 / N$ expansion, we find that Kondo peaks pinned to both Fermi surfaces exist to order $O(1 / N)$. A more rigorous analysis is needed to see whether the Kondo character found in the present treatment survives to infinite order.

Another more fundamental question is whether it is correct to assume that at low enough temperature a current carrying state in a mesoscopic system can be described using the Keldysh adiabatic perturbation technique as a nonequilibrium quantum coherent state as we consider here. The usual justification of such a treatment is that dissipation is absent in mesoscopic systems. A more detailed analysis of the thermodynamic meaning and consequences of the assumption can be found in Ref.
[18].

The author thanks P. A. Lee and N. S. Wingreen for many valuable discussions.

[1] P. L. McEuen et al., Phys. Rev. Lett. 66, 1926 (1991).

[2] A. T. Johnson et al., Phys. Rev. Lett. 69, 1592 (1992).

[3] U. Meirav, M. Kastner, and S. J. Wind, Phys. Rev. Lett. 65, 771 (1990).

[4] Y. Meir, N. S. Wingreen, and P. A. Lee, Phys. Rev. Lett. 66, 3048 (1991).

[5] T. K. Ng and P. A. Lee, Phys. Rev. Lett. 61, 1768 (1988).

[6] L. I. Glazman and M. E. Raikh, Pis'ma Zh. Eksp. Teor. Fiz. 47, 378 (1988) [JETP Lett. 47, 452 (1988)].

[7] P. A. Lee, Phys. Rev. Lett. 65, 2206 (1990).

[8] J. M. Kinaret, Y. Meir, N. S. Wingreen, P. A. Lee, and X. G. Wen, Phys. Rev. B 46, 4681 (1992).

[9] D. V. Averin and A. N. Korotkov, Zh. Eksp. Teor. Fiz. 97, 927 (1990) [Sov. Phys. JETP 70, 937 (1990)].

[10] S. Hershfield, J. H. Davies, and J. W. Wilkins, Phys. Rev. Lett. 67, 3720 (1991).

[11] Y. Meir, N. S. Wingreen, and P. A. Lee (to be published).

[12] For a review, see K. Chou, Z. Su, B. Hao, and Lu Yu, Phys. Rep. 118, 1 (1985).

[13] L. V. Keldysh, Zh. Eksp. Teor. Fiz. 47, 1515 (1964) [Sov. Phys. JETP 20, 1018 (1965)].

[14] C. Caroli, R. Combescot, P. Nozieres, and D. SaintJames, J. Phys. C 4, 916 (1971).

[15] For a review, see N. E. Bickers, Rev. Mod. Phys. 59, 845 (1987).

[16] O. Gunnarsson and K. Schönhammer, Phys. Rev. Lett. 50, 604 (1983).

[17] Y. Meir and N. S. Wingreen, Phys. Rev. Lett. 68, 2512 (1992).

[18] T. K. Ng, Phys. Rev. Lett. 68, 1018 (1992). 